\title{
Severe COVID-19 Associated With Liver Injury in Patients Without Preexisting Liver Disease
}

Abeer Altaf ${ }^{1}$, Zaigham Abbas ${ }^{1}$, Haider A. Mandviwalla ${ }^{2}$, Muhammad Ali Qadeer ${ }^{1}$, Mehreen Siyal ${ }^{1}$, Mahnoor Tariq $^{2}$, Asmara Ghafoor ${ }^{2}$, Muniba Karamat ${ }^{2}$, Bushra Shahid ${ }^{2}$, Mahnoor Ali ${ }^{2}$

1. Gastroenterology and Hepatology, Dr. Ziauddin Hospital, Karachi, PAK 2. Internal Medicine, Dr. Ziauddin Hospital, Karachi, PAK

Corresponding author: Abeer Altaf, abeeraltaf.98@gmail.com

\begin{abstract}
Objective: Coronavirus disease 2019 (COVID-19) is known to disturb liver function tests (LFTs). Not much literature is available regarding the effect of COVID-19 on LFTs in patients without preexisting liver disease. The study aimed to find the effect of COVID-19 in these patients.
\end{abstract}

Methods: This was a single-center, observational study with 142 patients who were admitted with COVID-19 during three months. Seven patients were excluded due to the presence of chronic liver disease.

Results: A total of 135 patients were included in the study aged between 18 and 95 years (mean $57.7 \pm 15.6$ ); among them, 93 were males (68.9\%). Hypertension was present in 74 patients (54.8\%), and diabetes was present in 48 patients (35.6\%). Fever was the chief complaint in 112 patients (83\%), followed by dyspnea in 93 patients (68.9\%) and cough in 79 patients (58.5\%). Elevated aspartate aminotransferase (AST) was seen in 35 patients (26\%), gamma-glutamyl transferase (GGT) in 43 patients (32\%), alanine transaminase (ALT) in 18 patients (24\%), alkaline phosphatase in 19 patients (14\%), bilirubin in six patients (4\%), and low albumin in 27 patients (20\%). Severe COVID-19, when compared with mild to moderate disease, was associated with elevated AST > two-time upper limit normal $(2 \mathrm{ULN})(\mathrm{p}=0.002)$, GGT $>2 \mathrm{ULN}(0.026)$, and lower albumin $(\mathrm{p}=$ $0.020)$, higher systemic inflammatory response syndrome (SIRS) (0.045), raised procalcitonin $(\mathrm{p}=0.045)$, higher ferritin $(\mathrm{p}=0.005)$, lower $\mathrm{pO} 2(\mathrm{p}=0.044)$, and higher Sequential Organ Failure Assessment score (SOFA) $(p=0.002)$ pointing to the inflammatory response as cause of liver injury. Factors predicting mortality with COVID-19 were assessed, which showed that direct bilirubin $(p=0.001)$, low albumin $(p=$ $0.013)$, tachypnea (0.002), and leukocytosis $(<0.001)$ were independently associated with increased COVID19-related mortality.

Conclusion: Patients suffering from COVID-19 have evidence of liver injury, which appears to be secondary to an inflammatory response that correlates with the severity of COVID-19.

Review began 04/03/2021 Review ended 04/23/2021 Published 04/26/2021

\section{○ Copyright 2021}

Altaf et al. This is an open access article distributed under the terms of the Creative Commons Attribution License CC-BY 4.0., which permits unrestricted use, distribution, and reproduction in any medium, provided the original author and source are credited.
Categories: Family/General Practice, Gastroenterology, Infectious Disease

Keywords: covid-19, liver function tests, ast (aspartate aminotransferase), severity of disease, liver injury

\section{Introduction}

The eruption of coronavirus disease-19 (COVID-19) is of great concern globally and has become a substantial challenge for physicians and public health authorities alike [1]. It has affected more than 100 million people worldwide and has been the cause of death of more than two million infected individuals. In Pakistan alone, the number of confirmed cases has surpassed 700,000 [2], crossing a death toll of over 11,000. Although when compared to other countries around the world the number of death is not as high, likely due to the immediate precautions taken by the country's government from an early stage and the possibility of a large number of infected individuals being asymptomatic [3], the number is still significant enough to be seen as a dangerous amount. The virus's rapid spread across the globe has largely been due to its alarming rate of infection, primarily through respiratory droplets and secondarily through physical contact [4]. Its usual clinical manifestations in the general population are a dry cough, fever, and flu-like symptoms, which can develop into acute respiratory distress syndrome and multiple system organ failures $[5]$.

Multiple studies have indicated that there is a progression of deranged liver functions in the infected individuals with COVID-19 previously diagnosed to have liver disease [6]. Fewer studies are showing the deteriorating effects of liver function in those without any preexisting liver-related illness. Therefore, it is essential to further investigate these changes in individuals who do not have any prior history of acute or chronic liver disease to better understand the development of abnormal liver functions during the period of infection.

To investigate this further, we documented the derangement of liver function tests (LFTs) among the patients admitted with COVID-19, followed each case, seeing parameters from inflammatory markers to 


\section{Cureus}

renal functions, coagulopathy, type of admission ward versus ICU, treatment provided, and outcomes.

\section{Materials And Methods}

The study was conducted at Dr. Ziauddin Hospital Clifton Campus, Karachi, Pakistan. This was a singlecenter, observational study. Patients $(n=142)$ with positive nasopharyngeal COVID-19 PCR for three months were included. Seven patients who had a previous history of chronic liver disease were excluded from the study, leaving a final number of 135 . The patients were then followed including the baseline labs, comorbidities, disease course, treatment provided, liver enzymes derangements, and the associations with severity of COVID-19, complications, and outcomes. Disease progress was graded by calculating Sequential Organ Failure Assessment (SOFA) Score, Chronic Liver Failure-Sequential Organ Failure Assessment (CLIFSOFA) Score, Acute Physiology and Chronic Health Evaluation II (APACHE II) Score, and the number of organ failures. The study was conducted after getting institutional ethics committee approval [2260620ZAGE].

COVID-19 severity was defined as mild, moderate, and severe according to the parameters shown in Table 1 .

\begin{tabular}{|c|c|c|}
\hline Mild & Moderate & Severe \\
\hline $\mathrm{No}_{2}$ requirement > $\mathrm{SaO}_{2} 93 \%$ & $\mathrm{O}_{2}$ requirement up to $10 \mathrm{~L}$ for $\mathrm{SsO}_{2} 93 \%$ & $\mathrm{O}_{2}$ requirement $>10 \mathrm{~L}$ for $\mathrm{SaO}_{2} 90 \%$ and $\mathrm{RR}>30$ \\
\hline No dyspnea & Dyspnea with <50\% of lung involvement & Dyspnea with $>50 \%$ of lung involvement \\
\hline No multisystem involvement & No multisystem involvement & Multiorgan dysfunction \\
\hline D-Dimer < 500 ng/ml FEU & D-Dimer 500-1000 ng/ml FEU & D-Dimer > 1000 ng/ml FEU \\
\hline CRP < 5 mg/L & CRP 5-10 mg/L & $\mathrm{CRP}>10 \mathrm{mg} / \mathrm{L}$ \\
\hline Ferritın < 500 ng/ml & Ferritın 500-1000 ng/ml & Ferritın > $1000 \mathrm{ng} / \mathrm{ml}$ \\
\hline NLR=1 & $\mathrm{NLR}<3$ & NLR $>3$ \\
\hline No secondary infection & No secondary infection & Evidence of secondary infection \\
\hline No ARDS & ARDS SpO $2 / \mathrm{HIO}_{2} 200-300$ & ARDS $<200$ \\
\hline No shock/need for Inotropes & No shock/need for inotropes & Shock/need for Inotropes \\
\hline
\end{tabular}

\section{TABLE 1: COVID severity}

$\mathrm{O}_{2}$, Oxygen; $\mathrm{SaO}_{2}$, oxygen saturation; CRP, C-reactive protein; FEU, fibrinogen equivalent units; NLR, neutrophil-lymphocyte ratio; ARDS, acute respiratory distress syndrome.

Continuous variables were expressed as mean and standard deviation, and analysis was performed by MannWhitney U test. Categorical data were analyzed using Fisher's exact test. A p-value of $<0.05$ was considered statistically significant. The statistical analysis was done on the IBM SPSS software version 20.0 (IBM Corp., Armonk, New York).

Acute liver injury was defined as a patient with any one of the following [7]:

i. Jaundice, with total bilirubin $>3 \mathrm{mg} / \mathrm{dl}$,

ii. Liver enzymes, alanine transaminase (ALT), aspartate aminotransferase (AST), gamma-glutamyl transferase (GGT), and alkaline phosphatase (ALP) > two-time upper limit normal (2xULN),

iii. International normalized ratio (INR) > 1.5, with no previous history of liver dysfunction.

\section{Results}

A total of 135 patients of age between 18 and 95 years (mean $=57.68)$ were included among which 93 were males (68.9\%) and 42 were females (31.1\%); 107 patients $(79.25 \%)$ improved and were discharged home, and 28 patients died (20.7\%). Of the 135 patients, 57 had mild symptoms (42.22\%), 32 had moderate symptoms (23.7\%), and 46 (34.07\%) suffered from severe COVID-19. The mean age of individuals affected with severe COVID-19 was 60.70 , and the mean age of individuals affected by mild to moderate disease was 55.38 . Mortality was highest in individuals admitted with severe COVID symptoms who required critical care (92.85\% vs $7.14 \%$ ) 
Among the comorbid conditions in the patients, hypertension was present in 74 patients (54.8\%), diabetes was present in 48 patients (35.6\%), cardiovascular disease was present in 25 patients (18.5\%), chronic kidney disease was present in seven patients (5.2\%), chronic obstructive pulmonary disease was present in six patients (4.4\%), and neurologic diseases were present in five patients (3.7\%). Of the study population, seven (5.2\%) were smokers, four of them had a history of alcohol intake (3\%), and obesity was present in six (4.4\%) individuals. Preexisting drug history included beta-blockers in 21 patients (15.6\%), diuretics in 35 patients (25.9\%), angiotensin-converting enzyme inhibitors in 31 patients (23\%), nonsteroidal anti-inflammatory drugs (NSAIDs) in eight patients (5.9\%), and statins in 20 patients $(14.9 \%)$.

The chief complaint of the majority of individuals (112 patients) admitted with COVID-19 was fever (82.96\%), followed by shortness of breath in 93 patients $(68.9 \%)$, cough in 79 patients $(58.5 \%)$, body aches in 27 patients (20\%), nausea in 10 patients $(7.4 \%)$, vomiting in nine patients $(6.7 \%)$, sore throat in seven patients (5.2\%), and two individuals presented with hematemesis (1.5\%). The presence of clinical or laboratory-proven jaundice was seen in one patient $(0.7 \%)$.

Liver enzyme derangements were seen in $32 \%$ of patients admitted and treated for COVID-19 and correlated with disease severity and outcome as evident in Table 2. AST levels ranged from 13 to 1883, a mean of $107.47 \pm 212.70$. Levels more than two times the ULN were seen in 23 patients with severe COVID-19 $(\mathrm{p}=$ 0.002). ALT levels ranged from seven to 1762 , a mean of $95.1 \pm 199.62$. Levels more than two times the ULN were seen in 16 individuals with severe COVID-19 ( $\mathrm{p}=0.096)$. GGT levels were between 12 and 964 with a mean of $121.24 \pm 135.24$. Levels of $2 x U L N$ were seen in 28 individuals suffering from severe COVID-19 $(\mathrm{p}=$ 0.026). Total bilirubin ranged between 0.2 and 15.2 with a mean of $0.95 \pm 1.72$. Values above $2 \mathrm{mg} / \mathrm{dl}$ were present in three patients with severe disease $(\mathrm{p}=0.302)$. ALP values were between 11 and 249 with a mean of $91.59 \pm 44.54$. ALP more than 120 were seen in 10 patients with severe disease $(\mathrm{p}=0.094)$.

\begin{tabular}{|c|c|c|c|c|c|}
\hline \multirow{2}{*}{ Liver Function Tests } & \multicolumn{2}{|c|}{ Mild to Moderate COVID-19 } & \multicolumn{2}{|l|}{ Severe COVID-19 } & \multirow{2}{*}{ P-value } \\
\hline & Mean \pm SD & Range & Mean \pm SD & Range & \\
\hline Total bilirubin (mg/dl) & $0.675 \pm 0.42$ & $0.2-2.4$ & $1.41 \pm 2.73$ & $0.3-15.2$ & 0.120 \\
\hline ALT (U/L) & $86.56 \pm 213.06$ & $7-1762$ & $110.05 \pm 175.21$ & $7-1061$ & 0.116 \\
\hline AST (U/L) & $93.22 \pm 218.61$ & $15-1883$ & $131.65 \pm 202.49$ & $13-1288$ & 0.022 \\
\hline GGT (IU/L) & $103.65 \pm 103.2$ & $12-586$ & $150.70 \pm 173.84$ & $14-969$ & 0.041 \\
\hline ALP (U/L) & $88.41 \pm 42.09$ & $11-249$ & $96.98 \pm 48.45$ & $35-220$ & 0.058 \\
\hline Albumin (g/dl) & $3.82 \pm 0.47$ & $2.5-4.7$ & $3.49 \pm 0.69$ & $1.9-4.6$ & 0.002 \\
\hline INR & $1.18 \pm 0.20$ & $0.9-2.0$ & $1.35 \pm 0.41$ & $0.9-2.7$ & 0.042 \\
\hline Elevated ALT $\geq 2 x U L N$ & $43 \pm 50.91$ & $7-79$ & $570.5 \pm 693.67$ & $80-1061$ & 0.096 \\
\hline Elevated AST $\geq 2 x U L N$ & $47 \pm 45.25$ & $15-79$ & $684 \pm 604$ & $80-1288$ & 0.002 \\
\hline Elevated GGT $\geq 2 x U L N$ & $45.5 \pm 47.37$ & $12-79$ & $524.5 \pm 628.61$ & $80-969$ & 0.026 \\
\hline Albumin $<3.5 \mathrm{~g} / \mathrm{dl}$ & $4.105 \pm 0.84$ & $3.51-4.7$ & $2.695 \pm 1.124$ & $1.9-3.49$ & 0.002 \\
\hline
\end{tabular}

\section{TABLE 2: LFTs in Severe COVID}

ALT, Alanine transaminase; AST, aspartate aminotransferase; GGT, gamma-glutamyl transferase; ALP, alkaline phosphatase; INR, international normalized ratio; 2xULN, two-time upper limit normal; LFT, liver function test.

Derangement of AST with more than two times ULN (>80) was compared with both mild/moderate and severe COVID as shown in Table 3. AST $>80$ was significant for higher severe inflammatory response syndrome (SIRS) score $1.29 \pm 0.463$ vs $1.59 \pm 0.503(p=0.045)$, raised procalcitonin $0.07 \pm 2.15$ vs $9.24 \pm 21.12$ $(p=0.045)$, raised ferritin $1008.14 \pm 1327.92$ vs $2354.89 \pm 2567.03(p=0.005)$, decreased oxygen in arterial blood gases on admission $91.75 \pm 33.91$ vs $71.77 \pm 32.88(\mathrm{p}=0.044)$, higher SOFA score $1.72 \pm 1.52$ vs $4.0 \pm$ $2.50(p=0.002)$, and increased number of organ damage $0.88 \pm 0.69$ vs $1.75 \pm 0.86(p=0.006)$. Outcomes of the patients were also monitored and showed that 51 patients with AST $<80$ improved and 12 died. In patients with AST > 80, 22 were improved and sent home, whereas 16 passed away $(\mathrm{p}=0.021)$. 


\section{Cureus}

\begin{tabular}{|c|c|c|c|c|c|}
\hline \multirow{2}{*}{ Variable } & \multicolumn{2}{|l|}{ AST $<80$} & \multicolumn{2}{|l|}{ AST $>80$} & \multirow{2}{*}{ P-value } \\
\hline & Mean & SD & Mean & SD & \\
\hline Mean arterial pressure & 91.97 & 12.17 & 90.59 & 15.43 & 0.631 \\
\hline $\mathrm{PaO}_{2} / \mathrm{FiO}_{2}$ & 108.82 & 143.15 & 98.18 & 184.13 & 0.804 \\
\hline SIRS & 1.29 & 0.463 & 1.59 & 0.503 & 0.045 \\
\hline $\mathrm{Hb}$ & 12.17 & 2.38 & 13.41 & 1.52 & 0.01 \\
\hline WBC & 10.71 & 4.95 & 10.78 & 4.95 & 0.943 \\
\hline Neutrophils & 77.82 & 11.509 & 81.74 & 8.98 & 0.075 \\
\hline Lymphocytes & 15.57 & 9.994 & 13.18 & 8.037 & 0.213 \\
\hline Platelets & 244.03 & 121.80 & 209.83 & 92.88 & 0.127 \\
\hline Creatinine & 1.37 & 1.15 & 1.27 & 0.71 & 0.61 \\
\hline РCT & 0.97 & 2.15 & 9.24 & 21.12 & 0.045 \\
\hline CRP & 113.30 & 132.87 & 121.26 & 55.75 & 0.72 \\
\hline Lactate & 1273.89 & 2696.26 & 40.27 & 41.85 & 0.33 \\
\hline Ferritin & 1008.14 & 1327.92 & 2354.89 & 2567.03 & 0.005 \\
\hline D-Dimer & 1775.53 & 2600.94 & 2663.31 & 3385.07 & 0.150 \\
\hline $\mathrm{PaO}_{2}$ & 91.75 & 33.91 & 71.77 & 32.88 & 0.044 \\
\hline SOFA & 1.72 & 1.526 & 4.00 & 2.50 & 0.002 \\
\hline APACHE II & 8.44 & 5.56 & 10.43 & 7.01 & 0.340 \\
\hline No. of organ failure & 0.88 & 0.69 & 1.75 & 0.86 & 0.006 \\
\hline
\end{tabular}

\section{TABLE 3: AST > 2ULN and severity of COVID}

SIRS, Systemic inflammatory response syndrome; WBC, white blood cell; PCT, procalcitonin; CRP, C-reactive protein; SOFA, Sequential Organ Failure Assessment; APACHE II, Acute Physiology and Chronic Health Evaluation II; AST, aspartate aminotransferase; 2ULN, two-time upper limit normal.

COVID-19 severity itself was also studied, and it was seen that patients suffering from severe illness have higher white blood cell (WBC) levels $9.97 \pm 3.97$ vs $11.69 \pm 5.46(\mathrm{p}=0.04)$, neutrophilia $76.65 \pm 10.18$ vs 82.91 $\pm 9.21(\mathrm{p}=0.001)$, lymphopenia $16.62 \pm 8.99 \mathrm{vs} 11.8 \pm 7.47(\mathrm{p}=0.003)$, and hence a higher neutrophil to lymphocytic ratio. Patients also had higher creatinine $1.21 \pm 0.66$ vs $1.58 \pm 1.25$ ( $\mathrm{p}=0.023)$, and some even required renal replacement treatment. LFTs such as AST $65.60 \pm 51.23$ vs $130.63 \pm 207.14(\mathrm{p}=0.022)$ and GGT $94.15 \pm 80.88$ vs $156.02 \pm 176.36(\mathrm{p}=0.041)$ were also significantly deranged, and often patients developed hypoalbuminemia as well $3.89 \pm 0.44$ vs $3.57 \pm 0.503(\mathrm{p}=0.001)$. Labs like INR $1.15 \pm 0.169 \mathrm{vs} 1.28$ $\pm 0.29(\mathrm{p}=0.042)$ and $\mathrm{D}$-dimers $1093.93 \pm 1397.4 \mathrm{vs} 3001.3 \pm 3824.8(\mathrm{p}=0.003)$ also showed substantial derangements. Prognostic markers of severity such as procalcitonin, lactate, C-reactive protein (CRP), and ferritin were also significantly elevated among the severely affected population with ferritin being statistically significant among all $1047 \pm 1258.46$ vs $17779.67 \pm 1931.84$ ( $\mathrm{p}=0.017)$. Disease severity calculators such as APACHE II Score $5.33 \pm 2.24$ vs $12.83 \pm 6.95(\mathrm{p}<0.001)$ and SOFA Score $1.5 \pm 1.15$ vs $4.3 \pm$ $2.43(\mathrm{p}<0.001)$ were also higher in severe patients as compared to mild to moderate ones. Arterial oxygen levels were also low in these patients on admission $89.62 \pm 9.81$ vs $93.28 \pm 3.86(\mathrm{p}=0.002)$, and they hence required supplemental oxygen via face mask, high-flow nasal cannula (HFNC), noninvasive ventilation (NIV), or ventilator.

Factors predicting mortality with COVID-19 were also assessed as mentioned in Table 4. Stepwise linear regression analysis of continuous variables showed four variables such as direct bilirubin $(\mathrm{p}=0.001)$, low albumin $(p=0.013)$, increased respiratory rate $(0.002)$, and raised WBC count $(<0.001)$, which were independently associated with increased COVID-19-related mortality. 


\section{Cureus}

\begin{tabular}{|c|c|c|c|}
\hline \multirow[t]{2}{*}{ Variable } & Alive & Dead & \multirow[t]{2}{*}{ P-value } \\
\hline & Mean/N (SD/\%) & Mean/N (SD/\%) & \\
\hline Age & $55.96(15.91)$ & $63.30(12.85)$ & 0.21 \\
\hline Mean arterial pressure & $91.59(13.863)$ & $87.00(17.75)$ & 0.103 \\
\hline Respiratory rate & $22.75(3.55)$ & $25.59(6.09)$ & 0.002 \\
\hline $\mathrm{SpO}_{2}$ & $93.05(5.79)$ & $87.28(11.13)$ & $<0.001$ \\
\hline TLC & $9.82(4.30)$ & $13.37(5.28)$ & $<0.001$ \\
\hline Neutrophils & $77.44(11.06)$ & $83.89(8.29)$ & 0.005 \\
\hline Urea & $48.32(47.31)$ & $60.07(44.46)$ & 0.033 \\
\hline Creatinine & $1.16(0.81)$ & $1.87(1.43)$ & 0.001 \\
\hline Total bilirubin & $0.63(0.39)$ & $1.80(3.20)$ & 0.001 \\
\hline Direct bilirubin & $0.29(0.196)$ & $1.19(2.44)$ & 0.001 \\
\hline ALT & 87.35 (199.209) & $117.79(202.761)$ & 0.489 \\
\hline AST & 90.05 (201.79) & $157.40(237.87)$ & 0.136 \\
\hline GGT & $115.36(136.86)$ & $137.90(131.35)$ & 0.435 \\
\hline ALP & $87.71(42.04)$ & $102.70(50.13)$ & 0.113 \\
\hline INR & $1.18(0.18)$ & $1.44(0.47)$ & 0.020 \\
\hline PCT & $1.14(5.40)$ & $12.37(22.76)$ & $<0.001$ \\
\hline Fasting blood sugar & 156.65 (74.67) & 113.45 (10.92) & 0.062 \\
\hline LDH & 864.41 (507.90) & 1207.72 (497.25) & 0.003 \\
\hline D-dimer & $1357.56(2240.19)$ & 3562.42 (3551.39) & $<0.01$ \\
\hline SOFA score & $1.89(1.39)$ & $5.11(3.19)$ & $<0.001$ \\
\hline APACHE II score & $6.50(3.85)$ & $14.17(6.44)$ & $<0.001$ \\
\hline No. of organ failure & $0.96(0.64)$ & $2.00(0.77)$ & 0.004 \\
\hline Fever & $92(82.14 \%)$ & 20 (17.85) & 0.117 \\
\hline Shortness of breath & 74 (79.56\%) & $19(20.43)$ & 1.00 \\
\hline Cough & $67(84.18 \%)$ & 12 (15.18\%) & 0.298 \\
\hline Presence of clinically significant jaundice & $0(0 \%)$ & $1(100 \%)$ & 0.043 \\
\hline Severe COVID-19 & $20(41.67 \%)$ & $28(58.33 \%)$ & $<0.001$ \\
\hline Albumin $<3.5$ & $22(73.68 \%)$ & $16(42.10 \%)$ & 0.013 \\
\hline AST > 80 & $57(82.60 \%)$ & 12 (17.39\%) & 0.009 \\
\hline Acute kidney injury & $5(27.77 \%)$ & $13(72.22 \%)$ & $<0.001$ \\
\hline Respiratory failure & $14(42.42 \%)$ & $19(57.57 \%)$ & $<0.001$ \\
\hline Derangement in LFT & 9 (56.25\%) & $7(43.75 \%)$ & 0.043 \\
\hline Hemodynamic shock & $1(6.25 \%)$ & $15(93.75 \%)$ & $<0.001$ \\
\hline DIC & $10(58.82 \%)$ & $7(41.17 \%)$ & 0.059 \\
\hline
\end{tabular}

\section{TABLE 4: Factors associated with mortality}

TLC, Total leucocyte count; ALT, alanine transaminase; AST, aspartate aminotransferase; GGT, gamma-glutamyl transferase; ALP, alkaline phosphatase; INR, international normalized ratio; PCT, procalcitonin; LDH, lactate dehydrogenase; 2xULN, two-time upper limit normal; LFT, liver function test; DIC, disseminated intravascular coagulation. 


\section{Discussion}

Acute liver injury is defined as a derangement in LFTs due to an acute insult in a previously healthy liver. Many studies have reported patients developing liver injury following COVID-19 infection [7-9]. Reasons for this liver injury are still unsure, but multiple studies show it may be due to the expression of angiotensinconverting enzyme II (ACE-II), which is identified as the main receptor for COVID-19 entry into cells. This could suggest that COVID-19 could have a direct effect on the hepatic system along with a sequalae to the ongoing inflammatory response $[10,11]$. This could also be due to the difference in population and how ACEII expression analysis differs between ethnicities [12], but further studies are required. Another hypothesis suggests immune-mediated liver injury, i.e., cytokine storm, causing the release of multiple proinflammatory cytokines particularly IL-6 that causes both pulmonary and extrapulmonary damages including the liver [8]. Patients affected with severe disease often have hypoxia and hypotension, and that might also be a causative factor to cause acute liver injury [13]. Lastly, many of the critically ill patients are on a variety of medications and parenteral nutrition, which may also contribute to the ongoing liver insult. All of these are the most commonly highlighted reasons for causing both direct and indirect hepatic damage in COVID-19-affected population.

As hypertension was the most common comorbidity in patients within our study, drug history might be significant in being a cause of worsening deranged liver enzyme levels. The three most common medications patients were using before admission were diuretics (25.9\%), angiotensin-converting enzyme (ACE) inhibitors (23\%), and beta-blockers (15.6\%), followed by statins (14.9\%). There is not enough evidence to prove whether the indication of using these drugs before hospitalization is truly significant to deranging liver enzymes [14].

The mean values of LFTs were above the normal range as seen within this study (Table 2). The liver function enzyme levels of patients with severe cases of COVID-19 were more deranged as compared to the liver enzyme levels of patients with mild or moderate cases. These enzymes, when elevated together, are strong indicators of liver injury. Total bilirubin (TB) and ALP levels were elevated in only a few cases, but overall remained under the normal ranges (mean $\mathrm{TB}=0.95$, mean ALP $=91.59$ ). These markers in numerous studies have had different results, some in which TB is markedly increased $[15,16]$ and others that coincide with our study [17]. Serum albumin levels were also not severely affected, but a mean decrease was seen in severe cases $(\mathrm{Alb}=3.49)$, which is closer to the lower normal limit as compared to the mild/moderate cases $(\mathrm{Alb}=$ 3.82 ).

AST levels greater than 80 showed more deranged infective markers typically seen in COVID-19 cases as shown in Table 3 . AST $>80$ showed severely deranged results (mean lactate $=2696.26$, mean D-dimer $=$ 2663.31, mean ferritin $=2354.89$, mean PCT $=9.24$ ). Although these markers were not as severely deranged in patients with AST $<80$, they were deranged nonetheless, showing how this presence of injury to the liver could be secondary to an inflammatory response. This has been seen in multiple studies within our subcontinent [17] and across the globe [18].

SOFA and APACHE II scores were also calculated to assess the prediction of mortality and to cross-reference with the severity of liver enzyme derangement. In severe cases, SOFA and APACHE II scores were markedly raised (mean SOFA $=4.33$, mean APACHE II $=12.83$ ), almost three times higher as compared to the scores calculated in mild/moderate cases. These calculations, including the significant increase in infective markers and LFTs, may show an overall systemic effect by the COVID-19 virus and may indicate a positive correlation between an increase in viral, liver injury, and inflammatory response [15].

In our study, COVID-19 mortality was associated with four biomarkers: serum albumin, direct bilirubin, total leucocyte count, and respiratory rate. Serum albumin has been proven to be an acute phase reactant. Under conditions of increased oxidative stress, it can undergo irreversible oxidation leading to increased cellular injury [19]. Patients who were severely affected, admitted to ICU, and those who required ventilatory support reported to have lower serum albumin levels than the mild to moderately affected population ( $3.37 \mathrm{vs} 3.83, \mathrm{p}$ $=0.013$ ). Bilirubin levels are reflective of acute liver injury, and direct bilirubin was increased among the nonsurvivors of our study population ( $0.29 \mathrm{vs} 1.19 \mathrm{p}=0.001)$. Chai et al. [20] showed that the expression of ACE-2 receptors on cholangiocytes was equivalent to that of the type II alveolar cells; however, the number is 20 times less in the hepatocytes. A study done in China by Liu et al. also showed that total bilirubin (HR: 9.45, 95\% confidence interval (CI): 2.21-40.47, $\mathrm{p}=0.002$ ), direct bilirubin levels (HR: 4.38 , 95\% CI: 1.78 $16.29, \mathrm{p}=0.03$ ), and conjugated bilirubin/unconjugated bilirubin (CB/UCB) (HR: $2.49,95 \%$ CI: 1.32-4.71, $\mathrm{p}=$ 0.01 ) were higher in the nonsurviving group [21] signifying toward an important mechanism of acute liver injury. COVID-19 being an infection triggers the inflammatory cascade and cytokines such as IL-6, IL-17, and TH-17, leading to SIRS, ARDS, and multiorgan failure. Hence the reason why increased total leucocyte count was seen as a marker influencing mortality $(9.82$ vs $13.37 \mathrm{p}<0.001)$. A study done in Wuhan, China, by Feng et al. also showed significant concordance between mortality and leukocytosis ( $\mathrm{HR}=1.14,95 \% \mathrm{CI}$ : $1.09-1.20, \mathrm{p}<0.001$ ) [22]. As patients commonly complain about shortness of breath, patients more severely affected with COVID-19 had higher incidences of respiratory failure as suggested by the higher respiratory rate $(22.75$ vs $25.59, \mathrm{p}=0.002)$, which was reflective of higher mortality in our study. Many studies globally 
showed similar results as well [23].

There are several strengths to this study. First, this study reflects the association between liver derangement and COVID-19 severity, as seen by the deranged LFTs along with COVID-19 inflammatory markers. Second, in this study by removing patients with preexisting and/or newly diagnosed chronic liver disease, we were able to see how COVID-19 affects naïve liver and see the clinical course. Lastly, this study also substantiates the use of AST, GGT, and albumin as COVID-19 severity markers as well as albumin and direct bilirubin as mortality markers along with raised leucocyte count and tachypnoea.

The limitations of this study are as follows: a sample size of 135 cases is smaller as compared to other studies that have been conducted but is still relevant to show a distinct derangement in liver enzyme levels.

Furthermore, the cases were all from a single hospital in a certain demographic of Pakistan, which may not show a proper representation of the disease and its effect on liver enzymes on a country-wide basis. Lastly, the laboratory results that were used in this study were results seen on admission, and so the effect of the progress on said laboratory results during a hospital stay has not been utilized.

\section{Conclusions}

In conclusion, COVID-19 has multisystemic effects. Liver injury caused by COVID-19 stems majorly from inflammatory response due to cytokines. The degree of liver damage is reflective of the severity and increased mortality, hence showing evidence that liver function enzymes have the potential to be utilized as surrogates to dictate the prognosis of COVID-19 severity.

\section{Additional Information \\ Disclosures}

Human subjects: Consent was obtained or waived by all participants in this study. Animal subjects: All authors have confirmed that this study did not involve animal subjects or tissue. Conflicts of interest: In compliance with the ICMJE uniform disclosure form, all authors declare the following: Payment/services info: All authors have declared that no financial support was received from any organization for the submitted work. Financial relationships: All authors have declared that they have no financial relationships at present or within the previous three years with any organizations that might have an interest in the submitted work. Other relationships: All authors have declared that there are no other relationships or activities that could appear to have influenced the submitted work.

\section{Acknowledgements}

I would like to thank the Department of Internal Medicine and Infectious Diseases of Dr. Ziauddin Hospital for helping us with the initial idealization, data sharing, and data entry of the study and for providing us free access to the COVID-19 patient directory needed for data entry.

\section{References}

1. Sultan S, Lim JK, Altayar O, et al.: AGA rapid recommendations for gastrointestinal procedures during the COVID-19 pandemic. Gastroenterology. 2020, 159:739-758. 10.1053/j.gastro.2020.03.072

2. COVID-19 Health Advisory Platform by Ministry of National Health Services Regulations and Coordination . (2020). Accessed: December 15, 2020: https://covid.gov.pk/.

3. Nisar MI, Ansari N, Khalid F, et al.: Serial household sero-surveys for COVID-19 in low and high transmission neighborhoods of urban Pakistan [IN PRESS]. medRxiv. 2020, 10.1101/2020.07.28.20163451

4. Li Q, Guan X, Wu P, et al.: Early transmission dynamics in Wuhan, China, of novel coronavirus-infected pneumonia. N Engl J Med. 2020, 382:1199-207. 10.1056/NEJMoa2001316

5. Huang C, Wang Y, Li X, et al.: Clinical features of patients infected with 2019 novel coronavirus in Wuhan, China. Lancet. 2020, 395:497-506. 10.1016/S0140-6736(20)30183-5

6. Increased hospitalization and mortality in patients with COVID-19 and pre-existing liver disease . (2020). Accessed: December 15, 2020: https://journalsblog.gastro.org/?p=8814.

7. Sarin SK, Choudhury A, Lau GK, et al.: Pre-existing liver disease is associated with poor outcome in patients with SARS CoV2 infection; The APCOLIS Study (APASL COVID-19 Liver Injury Spectrum Study). Hepatol Int. 2020, 14:690-70. 10.1007/s12072-020-10072-8

8. Ghoda A, Ghoda M: Liver injury in COVID-19 infection: a systematic review . Cureus. 2020, 12:e9487. 10.7759/cureus. 9487

9. Singh S, Khan A: Clinical characteristics and outcomes of coronavirus disease 2019 among patients with preexisting liver disease in the United States: a multicenter research network study. Gastroenterology. 2020, 159:768-771. 10.1053/j.gastro.2020.04.064

10. Yan R, Zhang Y, Li Y, Xia L, Guo Y, Zhou Q: Structural basis for the recognition of SARS-CoV-2 by fulllength human ACE2. Science. 2020, 367:1444-8. 10.1126/science.abb2762

11. Xu L, Liu J, Lu M, Yang D, Zheng X: Liver injury during highly pathogenic human coronavirus infections . Liver Int. 2020, 40:998-1004. 10.1111/liv.14435

12. Cao Y, Li L, Feng Z, et al.: Comparative genetic analysis of the novel coronavirus (2019-nCoV/SARS-CoV-2) receptor ACE2 in different populations. Cell Discov. 2020, 6:11. 10.1038/s41421-020-0147-1

13. Chand N, Sanyal AJ: Sepsis-induced cholestasis. Hepatology. 2007, 45:230-41. 10.1002/hep.21480

14. Zhang C, Shi L, Wang FS: Liver injury in COVID-19: management and challenges . Lancet Gastroenterol 


\section{Cureus}

Hepatol. 2020, 5:428-30. 10.1016/S2468-1253(20)30057-1

15. Saini RK, Saini N, Ram S, et al.: COVID-19 associated variations in liver function parameters: a retrospective study. Postgrad Med J. 2020, 10.1136/postgradmedj-2020-138930

16. Paliogiannis P, Zinellu A: Bilirubin levels in patients with mild and severe Covid-19: a pooled analysis . Liver Int. 2020, 40:1787-8. 10.1111/liv.14477

17. Asghar MS, Akram M, Rasheed U, et al.: Derangements of liver enzymes in Covid-19 positive patients of Pakistan: a retrospective comparative analysis with other populations. Arch Microbiol Immunol. 2020, 4:110-120. 10.26502/ami.93650050

18. Hundt MA, Deng Y, Ciarleglio MM, Nathanson MH, Lim JK: Abnormal liver tests in COVID-19: a retrospective observational cohort study of 1,827 patients in a major U.S. hospital network. Hepatology. 2020, 72:1169-76. 10.1002/hep.31487

19. Violi F, Cangemi R, Romiti GF, et al.: Is albumin predictor of mortality in COVID-19? [IN PRESS] . Antioxid Redox Signal. 2020, 10.1089/ars.2020.8142

20. Chai X, Hu L, Zhang Y, et al.: Specific ACE2 expression in cholangiocytes may cause liver damage after 2019-nCoV infection [IN PRESS]. bioRxiv. 2020, 10.1101/2020.02.03.931766

21. Liu Z, Li J, Long W, et al.: Bilirubin levels as potential indicators of disease severity in coronavirus disease patients: a retrospective cohort study. Front Med (Lausanne). 2020, 7:598870. 10.3389/fmed.2020.598870

22. Feng X, Zhu B, Jiang C, et al.: Correlation between white blood cell count at admission and mortality in COVID-19 patients: a retrospective study [IN PRESS]. BMC Infect Dis. 2020, 10.21203/rs.3.rs-20383/v1

23. Bahl A, Van Baalen MN, Ortiz L, et al.: Early predictors of in-hospital mortality in patients with COVID-19 in a large American cohort. Intern Emerg Med. 2020, 15:1485-99. 10.1007/s11739-020-02509-7 